IdeAs

Idées d'Amériques

$7 \mid 2016$

Cinéma et histoire dans les Amériques

\title{
Cinéma et histoire dans les Amériques
}

Ignacio Del Valle Dávila and Eduardo Morettin

\section{(2) OpenEdition}

Journals

Electronic version

URL: https://journals.openedition.org/ideas/1503

DOI: 10.4000/ideas.1503

ISSN: 1950-5701

Translation(s):

Cinema and History in the Americas - URL : https://journals.openedition.org/ideas/3103 []

Cine e historia en las Américas - URL : https://journals.openedition.org/ideas/3113 [es]

Cinema e história nas Américas - URL : https://journals.openedition.org/ideas/3144 [pt]

\section{Publisher}

Institut des Amériques

\section{Electronic reference}

Ignacio Del Valle Dávila and Eduardo Morettin , "Cinéma et histoire dans les Amériques", IdeAs [Online], 7 | 2016, Online since 30 June 2016, connection on 19 October 2022. URL: http:// journals.openedition.org/ideas/1503; DOI: https://doi.org/10.4000/ideas.1503

This text was automatically generated on 19 October 2022

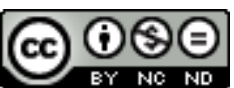

Creative Commons - Attribution-NonCommercial-NoDerivatives 4.0 International - CC BY-NC-ND 4.0 https://creativecommons.org/licenses/by-nc-nd/4.0/ 


\title{
Cinéma et histoire dans les Amériques
}

\author{
Ignacio Del Valle Dávila and Eduardo Morettin
}

1 Le cinéma est l'une des principales expressions artistiques et culturelles des $\mathrm{XX}^{\mathrm{e}}$ et XXI siècles. C'est aussi un puissant moyen de communication de masse. Ces caractéristiques en font une source primaire d'un grand intérêt tant pour l'étude des projets politiques que pour celles des imaginaires collectifs au sein de communautés et dans des périodes historiques déterminées. Pour cette raison, nombreux ont été les chercheurs à s'intéresser à la relation entre cinéma et histoire.

2 Le dossier "Cinéma et histoire dans les Amériques » vise à comprendre le rôle joué par le cinéma dans la représentation du passé historique de différents pays du continent américain. Dans le même temps, par cette étude, nous entendons présenter diverses propositions pratiques sur les manières d'utiliser le cinéma comme source d'étude de l'histoire. Par conséquent, les articles proposent des analyses qui touchent tant aux débuts du cinéma qu'à ses tendances récentes: le cinéma dit muet, le passage du langage visuel au langage audiovisuel, le développement du mélodrame et du film historique, le film d'auteur des années 1960, le cinéma militant des années 1960 et 1970, et les documentaires autobiographiques des vingt dernières années.

3 Il n'est pas possible, en ces quelques pages, de récapituler de manière exhaustive les principales contributions théoriques qui envisagent le cinéma depuis une perspective historique. Nous nous limiterons à signaler quelques points d'inflexion dans le développement du champ de recherche. D'une manière générale, on peut affirmer que les films documentaires comme les films de fiction ont mis des décennies à trouver leur légitimité en tant que source pour l'histoire, et ce malgré leur important taux de pénétration - surtout pour la fiction - en tant que divertissement de masse associé à sa double condition d'art et d'industrie. Ce n'est qu'à partir des travaux de Marc Ferro des années 1970, publiés sous forme d'ouvrage en 1977 dans Cinéma et histoire, que sont systématisés des principes méthodologiques sur l'utilisation du cinéma comme source par l'historien. D'après lui, l'analyse des images en mouvement permettrait de lancer une "contre-analyse de la société », en révélant des zones de réalité latentes, "des 
zones non visibles du passé des sociétés; révélant, par exemple, les autocensures et lapsus d'une société » (Ferro M., 2009: 27). Bien que cette théorie ait été amplement discutée et critiquée ces trente dernières années, la défense qu'il fait de la valeur de l'image en mouvement comme « document historique » et " agent de l'histoire » dans une société qui la produit et la consomme demeure pertinente pour la recherche des relations entre cinéma et histoire (Ferro M., 2009 : 17).

Ce champ d'études s'est rapidement enrichi de la parution des livres de Pierre Sorlin : Sociologie du cinéma, (Sorlin P., 1977), et The Film in History. Restaging the Past (Sorlin P., 1980). Sorlin, particulièrement attentif aux relations entre le cinéma de fiction historique et la discipline historique, a cherché à démontrer que la représentation du passé par le cinéma laisse entrevoir les questions politiques contemporaines. D'un autre côté, comme l'a lui-même reconnu Sorlin, son intérêt dans les années 1980 pour la sémiotique en tant que méthode d'approche de l'analyse filmique semble moins pertinent aujourd'hui.

$5 \mathrm{Au}$ cours de la dernière décennie du XXe siècle et durant les deux premières du $\mathrm{XXI}^{\mathrm{e}}$, les études associant cinéma et histoire se sont étendues et diversifiées. Au-delà d'une révision méthodologique, nombreux ont été les travaux qui se sont penchés sur l'analyse d'un film ou d'un contexte spécifique, et qui ont permis de vérifier la validité de ces principes généraux. Dans le contexte français, Michèle Lagny a interrogé le développement d'une méthodologie qui, sans abandonner l'analyse du texte filmique, considère indispensable à son interprétation l'insertion dans le contexte culturel et dans la tradition filmique, au-delà de la détermination des conditions politiques et économiques de la production (Lagny M., 2009). Dans une tout autre perspective, à partir du constat de la prolifération de "films en costumes ", Pierre Beylot et Raphaëlle Moine se sont intéressés depuis les années 1980 aux relations entre le film historique de fiction et la notion de patrimoine historique et culturel (Beylot P., et R. Moine, 2009). Sylvie Lindeperg, de son côté, a développé le concept de micro-histoire en mouvement pour expliquer sa manière d'aborder Nuit et brouillard (Alain Resnais, 1955). Son travail sur ce documentaire mobilise autant l'analyse des formes cinématographiques que l'étude minutieuse du contexte dans lequel il a été produit et diffusé (Lindeperg S., 2007 : 8). Antoine De Baecque, pour sa part, développe le concept d'« histoire-caméra ", par lequel il cherche à démontrer que le cinéma peut être considéré comme une forme d'histoire du fait de sa capacité à représenter le passé, à se transformer en archive du passé et à atteindre au moyen du montage de nouvelles formes de réflexion sur le passé reconstruit ou préservé sur la pellicule (De Baecque A., 2008 : 13).

Outre-Atlantique, les travaux de Robert Rosenstone ont eu une forte répercussion. Selon lui, les films historiques, y compris les fictions, modèlent notre conscience historique, conjointement à d'autres médias audiovisuels. Le principal objectif de Rosenstone est en effet d'enquêter sur la manière dont les films créent un monde historique (Rosenstone R., 2010). Dans sa tentative d'appréhender la construction d'un savoir sur le passé à travers le cinéma, l'auteur en vient à mettre sur le même plan le travail du cinéaste et celui de l'historien, bien que les règles d'interaction de leurs œuvres avec le passé soient différentes des règles de l'histoire écrite (Rosenstone R., 2010 : 22). La thèse de Rosenstone est qu'aussi bien l'histoire écrite que le cinéma sont des récits construits par convention au sein desquels il est possible de trouver des éléments imaginaires ou irréels. De cette manière, l'auteur insère la production 
universitaire tout comme les arts du spectacle dans une catégorie floue constituée de récits.

7 Il faut également observer le renouvellement dans le domaine de l'histoire du cinéma. À partir du Congrès de Brighton, tenue sous l'égide de la Fédération Internationale des Archives du Film en 1978, la proximité des questions apportées par l'histoire culturelle allait permettre la proposition d'une méthode d'analyse filmique incluant la dimension historique. Comme l'écrit Tom Gunning, l'un des participants :

Si les méthodes d'analyse des films comme systèmes de signification sont différentes de celles des films comme marchandise, elles ne s'excluent pas mutuellement et ne sont pas non plus en dernière instance indépendantes. [...] Nous devons développer des méthodes d'analyse des films eux-mêmes qui incluent une dimension historique [...] il est temps de mener une comparaison diachronique des films au sein de l'histoire (Gunning T., $1990: 86$ ).

Rosenstone, Ferro et Sorlin sont les principaux auteurs avec lesquels la recherche latino-américaine parvient à dialoguer. Cela peut s'expliquer, dans une certaine mesure, par la traduction de quelques-uns de leurs livres en portugais et en espagnol. Ce champ de recherche a commencé à se développer en particulier au Brésil, où depuis le milieu des années 1990, prolifère une littérature scientifique ${ }^{1}$. On peut mentionner les livres História e cinema (Capelato M. H., Morettin, E., Napolitano M. et al., 2007) ; Cinematógrafo: um olhar sobre a história (Nóvoa J., Biscouto Fressato S. et Feigelson K., 2009) ; História e Audiovisual, numéro spécial de la revue Estudos Históricos (Kornis M., 2012) ; le livre História e documentário (Morettin E., Napolitano M. et Kornis M., 2012) ; le dossier As imagens no tempo e os tempos da imagem de la revue Histórica, Questões \& Debates (Bastos Kern M. L. et Kaminski R., 2014). On peut également mentionner diverses monographies, comme Canibalismo dos fracos (Freire Ramos A., 2002), à propos du film Os Inconfidentes (Joaquim Pedro de Andrade, 1972), ou Humberto Mauro, Cinema, História (Morettin E., 2013), ainsi que de nombreux articles, journées d'études, congrès, séminaires et projets de recherche.

9 Tout film dialogue avec la société et l'époque où il a été réalisé. Il incorpore et mobilise des imaginaires et des idéologies d'une société en conflit, tout en ayant potentiellement une incidence sur eux - en tant qu'agent historique. Nous porterons donc une attention particulière à la manière dont les films étudiés contribuent à la construction d'imaginaires sociaux et à la défense de discours et de projets politiques. Afin d'aborder le cinéma comme source pour l'histoire, il convient de prendre en compte sa spécificité artistique, ainsi que les caractéristiques propres au langage audiovisuel. Sinon, nous risquerions de l'analyser à partir d'une méthodologie héritée de l'écrit qui s'avère inadaptée pour rendre compte du cinéma comme document de son temps. L'enjeu est, précisément, de reconnaître le caractère spécifique du champ cinématographique. Par conséquent, il convient de souligner l'importance de l'analyse filmique comme outil à la fois pour étudier des documents cinématographiques et pour établir les problématiques qui guideront la réflexion.

Les articles du dossier recouvrent trois zones géographiques - l'Amérique du Sud, les Caraïbes et l'Amérique du Nord - et quatre espaces linguistiques - hispanophone, lusophone, anglophone et francophone. Nous avons privilégié l'étude de cas nationaux pour éviter le risque d'une vision généraliste et sans grande profondeur de la problématique abordée. Néanmoins, dans les cas d'expériences cinématographiques concrètes en lien avec l'exil et la migration, nous avons considéré qu'il était nécessaire 
de ne pas suivre ces repères nationaux afin de proposer un rapprochement transnational, car l'objet-même l'exigeait.

11 Le dossier commence par le Brésil avec les articles d'Ismail Xavier, Eduardo Morettin et Fernando Seliprandy. Le premier étudie les relations entre allégorie historique et engagement dans les films du cinéaste Glauber Rocha (actif 1959-1980). Quant à Eduardo Morettin, il analyse les connexions entre discours historique et discours cinématographique dans la représentation de l'arrivée du colonisateur faite par Mauro à une époque où le gouvernement de Getúlio Vargas (1930-1945) était en quête d'une légitimation symbolique. Finalement, Fernando Seliprandy étudie les convergences et divergences entre deux documentaires qui traitent du guérillero Carlos Marighella: Marighella : retrato falado do guerrilheiro (Silvio Tendler, 2001) et Marighella (Isa Grinspum Ferraz, 2012).

Les films de fiction des années 1970 et 1980 font l'objet de deux articles sur le cinéma argentin. Dans le premier, Ignacio Del Valle Dávila analyse les relations entre le cinéma historique et le projet idéologique de la dictature dite "Révolution Argentine" (1966-1973), à partir du film El santo de la espada (Leopoldo Torre-Nilsson, 1970), à propos du héros de l'indépendance, José de San Martín (1778-1850). Ana Laura Lusnich, de son côté, étudie la prééminence d'allégories et de métaphores dans des films produits au cours de la dernière dictature argentine (1976-1983) et au début de la période post-dictatoriale (1983-1989), comme réflexion sur la terreur d'État et sur le contrôle social qui a caractérisé cette période. L'analyse de Lusnich se concentre sur les long-métrages Los miedos (Alejandro Doria, 1980) et Hay unos tipos abajo (Rafael Filipelli, Emilio Alfaro, 1985).

Mariana Villaça aborde la relation entre la politique culturelle du gouvernement cubain et l'influent Instituto Cubano del Arte e Industria Cinematrográfica (ICAIC), en montrant également les conflits internes entre la direction de l'institut et quelques cinéastes critiques à deux moments particulièrement tendus dans le champ culturel de l'île : 1968 et le début des années 1970. Les films Coffea Arábiga (Nicolás Guillén Landrián, 1968) et El otro Francisco (Sergio Giral, 1973) sont des objets privilégiés pour analyser ces relations.

Les articles d'Anne-Marie Paquet-Deyris, Sergio Alpendre et Vincent Souladié abordent des moments différents du cinéma des Etats Unis. Anne-Marie Paquet-Deyris étudie la représentation cinématographique du président Abraham Lincoln, à partir de trois films produits entre le début et la fin des années 1930 : Abraham Lincoln (D. W. Griffith, 1930), Vers sa destinée (Young Mr. Lincoln, John Ford, 1939) et Abraham Lincoln (Abe Lincoln in Illinois, John Cromwell, 1940). Les autres articles consacrés au cinéma hollywoodien étudient des relectures de ce que l'on appelle le rêve américain durant des moments de crise. Dans le premier, Sérgio Alpendre analyse les films Rocky (John G. Avildsen, 1976) et La fièvre du samedi soir (John Badham, 1977) qu'il considère comme des métaphores d'un moment de crise du capitalisme face au premier choc pétrolier. Enfin, Vincent Souladié fait une analyse critique des relations au niveau des images entre les films de super-héros produits après le 11 septembre 2001 et les attentats contre les tours jumelles de New York.

15 L'étude des relations entre cinéma et histoire en Amérique du Nord se referme sur l'analyse d'un film inséré dans l'espace linguistique francophone. Dans son article, Henri Gervaiseau analyse Le règne du jour (Pierre Perrault, 1967), en explorant la manière dont le déplacement entre des espaces sociaux et géographiques distants 
dessine un sentiment d'appartenance territoriale. Le dossier se ferme sur un article qui se rapproche d'expériences cinématographiques liées à la migration et à l'exil politique, avec un accent mis sur la circulation et les échanges transnationaux. Jennifer Cazenave aborde l'expérience de la migration et de l'exil à partir de l'étude de deux film-journals : Reminiscences of a Journey to Lithuania (Jonas Mekas, 1973) et Diary (David Perlov, 1973-1983).

L'ensemble du dossier propose une vision variée et complexe des relations entre cinéma et histoire sur le continent, à partir de cas spécifiques qui incluent aussi bien l'analyse de contextes nationaux que de circulations transnationales. Nous aimerions souligner que la perspective historique qui oriente ces études favorise une confluence productive entre des chercheurs du cinéma venus de différents horizons. La présence dans ce dossier de textes rédigés par des spécialistes en histoire, études cinématographiques, communication et esthétique marque cette orientation.

Le lecteur remarquera facilement que les approches et thématiques de bien des textes dialoguent entre eux. Ismail Xavier et Ana Laura Lusnich mettent l'accent sur l'importance de l'allégorie pour le cinéma; les narrations de fondation sont abordées par Eduardo Morettin, Ignacio Del Valle Dávila et Anne-Marie Paquet-Deyris; le filmessai par Jennifer Cazenave et Henri Gervaiseau ; le portrait hagiographique par AnneMarie Paquet-Deyris, Ignacio Del Valle Dávila et Fernando Seliprandy; les relations entre cinéma et projet révolutionnaire apparaissent chez Ismail Xavier et Mariana Villaça.

Malgré la multiplicité des problématiques abordées, tous les articles du dossier se caractérisent par la place centrale attribuée à l'analyse cinématographique pour la compréhension des phénomènes historiques au sein desquels s'inscrivent les œuvres étudiées. Nous n'avons pas jugé pertinent de réduire cette multiplicité de propositions à un nombre limité de thématiques. Il serait cependant possible de proposer quelques tendances générales. Toutes les contributions traitent directement de la relation entre art et politique, à différentes époques et sous différents formats. Dans ce sens, il est intéressant de relever que les travaux sur l'Amérique latine mettent l'accent sur l'apogée et sur la crise du cinéma engagé dans des projets révolutionnaires, ainsi que sur les relations plus ou moins tendues entre la production cinématographique et les gouvernements autoritaires. Pour ce qui est des articles sur les Etats-Unis, l'accent est plutôt sur la relation entre le développement du marché cinématographique, qui rend possibles des productions toujours plus spectaculaires depuis le début du XXe siècle, et la mise en place ou la révision de récits mélodramatiques des efforts et des ambitions de héros individuels. Ces récits sont liés à un imaginaire qui souscrit à la concurrence, la lutte pour l'ascension sociale et le succès, une caractéristique fondamentale de la société nord-américaine.

L'origine de ce dossier remonte aux derniers mois de 2013, lorsqu'une première proposition a été présentée à la revue IdeAs. Il s'agit d'une initiative liée au groupe de recherche « História e Audiovisual: circularidades e formas de comunicação » (Histoire et audiovisuel: circularités et formes de communication), coordonné par les professeurs Eduardo Morettin et Marcos Napolitano de l'Université de São Paulo. Dès le début, le choix a été fait d'intégrer des chercheurs venus d'autres espaces universitaires, afin de favoriser les échanges internationaux. Au-delà de l'attache institutionnelle, l'origine nationale des auteurs est particulièrement variée (brésilienne, française, argentine, franco-brésilienne, franco-américaine, espagnole- 
chilienne), ce qui peut être interprété comme le signe de l'internationalisation croissante de la recherche ${ }^{2}$. Au final, la pluralité des approches présentes dans ce dossier manifeste aussi la consolidation d'un champ d'étude tourné vers la réflexion entre le cinéma et les sciences humaines.

\section{BIBLIOGRAPHY}

Bastos Kern, Maria Lúcia et Kaminski, Rosane (org.), As imagens no tempo e os tempos da imagem, História Questões \& Debates, $n^{\circ}$ 61, 2014.

Beylot, Pierre et Moine, Raphaëlle (dir.), Fictions patrimoniales, sur grand et petit écran, Bordeaux, PUB, 2009.

Capelato, Maria Helena ; Morettin, Eduardo ; Napolitano, Marcos ; Saliba, Elias Thomé (org.), História e cinema, São Paulo, Alameda, 2007.

De Baecque, Antoine et Chevalier, Philippe, Dictionnaire de la pensée du cinéma, Paris, Puf, 2012.

De Baecque, Antoine, L'histoire-caméra, Paris, Gallimard, 2008.

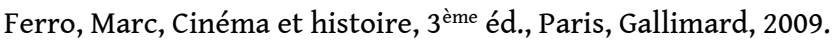

Freire Ramos, Alcides, Canibalismo dos fracos, Bauru, EDUSC, 2002.

Gunning, Tom, « Non-continuity, Continuity, Discontinuity. A Theory of Genres in Early Films », in Thomas Elsaesser (éd.), Space, Frame, Narrative, Londres, BFI Publishing, 1990.

« História e Audiovisual: circularidades e formas de comunicação » : http://

historiaeaudiovisual.weebly.com/teses-e-dissertaccedilotildees.html, page consultée le 26 mars 2016.

Kornis, Mônica Almeida (org.), História e Audiovisual, Estudos Históricos, vol. 26, n 51, 2013 , http://lisa.revues.org/index846.html, page consultée le 31 mars 2016.

Lagny, Michèle, «O cinema como fonte da história », in Jorge Nóvoa, Soleni Fressato et Kristian Feigelson, (dir.), Cinematógrafo: um olhar sobre a história, Salvador, EDUFBA; São Paulo, Edunesp, 2009, p. 99-132.

Lindeperg, Sylvie, « Nuit et brouillard » un film dans l'histoire, Paris, Odile Jacob, 2007.

Morettin, Eduardo ; Napolitano, Marcos ; Kornis, Mônica Almeida (org.), História e documentário, Rio de Janeiro, Editora FGV, 2012.

Morettin, Eduardo, Humberto Mauro, Cinema, História, São Paulo, Alameda, 2013.

Nóvoa, Jorge ; Fressato, Soleni ; Feigelson, Kristian (org.), Cinematógrafo: um olhar sobre a história, Salvador, EDUFBA; São Paulo, Edunesp, 2009.

Rosenstone, Robert, A história nos filmes, os filmes na história, traduit de l'anglais (États-Unis) par Marcello Lino, [éd. orig. History on Film/Film on History, Harlow, Pearson Education] São Paulo, Paz e Terra, 2010 [2006]. 
Sorlin, Pierre, Sociologie du cinéma : ouverture pour l'histoire de demain, Paris, Aubier Montaigne, 1977.

Sorlin, Pierre, The film in history : restaging the past, Oxford, Basil Blackwell, 1980.

\section{NOTES}

1. La liste de ces travaux est disponible sur le site web du groupe de recherche « Histoire et Audiovisuel: circularités et formes de communication »: http:// historiaeaudiovisual.weebly.com/teses-e-dissertaccedilotildees.html, page consultée le 26 mars 2016.

2. Soulignons dans ce sens la trajectoire du Professeur Ismail Xavier - dont l'article ouvre le dossier -, l'une des principales références internationales sur l'œuvre de Glauber Rocha et l'étude de l'allégorie dans le cinéma latino-américain, dont les travaux ont été largement traduits en Europe, aux Etats-Unis et en Amérique latine. Les contributions de Xavier ont donné lieu à une notice sur lui dans le Dictionnaire de la pensée du cinéma (De Baecque A. et Chevalier P., 2012).

\section{AUTHORS}

\section{IGNACIO DEL VALLE DÁVILA}

D’origine espagnole et chilienne, Ignacio Del Valle Dávila est docteur en cinéma (Université de Toulouse - Jean Jaurès), et a réalisé un post-doctorat en histoire à l'Université de São Paulo. Il est actuellement professeur de troisième cycle de l'Institut d'Art de l'Université de Campinas (Unicamp), où il poursuit ses recherches sur le cinéma latino-américain avec une bourse de postdoctorat CAPES. Auteur des livres Cámaras en trance: el nuevo cine latinoamericano, un proyecto cinematográfico subcontinental (Santiago du Chili, Cuarto Propio, 2014) et Le Nouveau Cinéma Latinoaméricain (1960-1974) (Rennes, PUR, 2015). Il est membre du groupe de recherche CNPq « História e Audiovisual: circularidades e formas de comunicação » et du conseil de rédaction de la revue Cinémas d'Amérique latine.

\section{EDUARDO MORETTIN}

Eduardo Morettin est professeur d'Histoire de l'Audiovisuel de l'École de Communications et des Arts de l'Université de São Paulo. Il est l'auteur de « Humberto Mauro, Cinema, História » et l'un des organisateurs de « História e Cinema: dimensões históricas do audiovisual » (2 ème éd., SP, Alameda Editorial, 2011), « História e Documentário » (RJ, FGV, 2012) et « Visualidades Hoje » (Salvador, Edufba, 2013). Membre du Conseil de la Cinémathèque Brésilienne depuis 2007, il est l'un des responsables du groupe de recherche « História e Audiovisual: circularidades e formas de comunicação » (site http://historiaeaudiovisual.weebly.com/). Il est chercheur CNPq (Prix de productivité niveau 2), ayant réalisé un post-doctorat à l'Université Paris I (2012). 